\title{
Exotisme, initiatives marchandes et pratiques alimentaires dans les commerces grecs de Bruxelles
}

Exotism, Commercial Activities and Food Choices in the Greek Shops in Brussels

\section{Katerina Seraïdari}

\section{CpenEdition}

\section{Journals}

Édition électronique

URL : https://journals.openedition.org/ceb/7115

DOI : $10.4000 /$ ceb. 7115

ISSN : 2261-4184

Éditeur

INALCO

Édition imprimée

ISBN : 978-2-85831-230-6

ISSN : 0290-7402

Référence électronique

Katerina Seraïdari, "Exotisme, initiatives marchandes et pratiques alimentaires dans les commerces grecs de Bruxelles », Cahiers balkaniques [En ligne], Hors-série | 2016, mis en ligne le 16 mars 2017, consulté le 06 juillet 2021. URL : http://journals.openedition.org/ceb/7115 ; DOI : https://doi.org/ $10.4000 /$ ceb.7115

Ce document a été généré automatiquement le 6 juillet 2021.

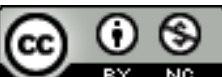

Cahiers balkaniques est mis à disposition selon les termes de la Licence Creative Commons Attribution - Pas d'Utilisation Commerciale 4.0 International. 


\title{
Exotisme, initiatives marchandes et pratiques alimentaires dans les commerces grecs de Bruxelles
}

\author{
Exotism, Commercial Activities and Food Choices in the Greek Shops in Brussels
}

\author{
Katerina Seraïdari
}

1 Afin d'attirer la main-d'œuvre nécessaire au fonctionnement de ses mines, la Belgique a conclu une convention bilatérale avec la Grèce en 1957. Les Grecs, en Belgique comme dans tous les pays où ils ont immigré, se sont ensuite spécialisés dans le domaine de l'alimentation, préférant au salariat le travail indépendant qui s'appuie sur une maind'œuvre familiale et des réseaux de solidarité intra-groupe. Cette orientation économique est liée à la désindustrialisation et à la hausse du chômage après la crise pétrolière des années 1970, ainsi qu'aux changements dans les pratiques alimentaires. En effet, après la fin de la Seconde Guerre mondiale, l'industrialisation de l'agroalimentaire et les techniques de conservation, de conditionnement et de transport, qui déconnectent pour la première fois l'aliment de son enracinement géographique et des contraintes climatiques, mettent fin à des siècles de malnutrition en Europe et assurent une grande diversité alimentaire. Un sentiment d'abondance s'installe progressivement dans les sociétés occidentales. Dans le même temps, l'aliment préparé devient une marchandise: en raison du travail féminin et de la redéfinition des rôles sociaux des sexes, la fonction culinaire diminue dans l'alimentation quotidienne. Le développement du secteur de l'alimentation hors du foyer fait basculer l'acte de manger de la sphère domestique au domaine économique, et de la cuisine à l'usine (Poulain, 2002). En somme, les premiers commerces que les Grecs ouvrent à Bruxelles sont le résultat concomitant de la désindustrialisation de la Belgique et de l'industrialisation grandissante de la sphère alimentaire.

2 Si la première révolution consumériste a eu lieu en Europe "à l'époque de la Renaissance grâce à la demande conjuguée de l'aristocratie traditionnelle et de la bourgeoisie ascendante ", la seconde se définit par «l'avènement de la consommation de masse » et la troisième, celle qui nous intéresse ici, par la consommation croissante 
de produits exotiques (RAULIN, 2000,14). Ce consumérisme de l'exotique nourrit le sentiment d'abondance qui caractérise les sociétés occidentales. Les initiatives commerciales grecques que nous examinerons ici s'inscrivent dans le registre d'une cuisine méditerranéenne qui semble être plus «pittoresque » qu'exotique (RÉGNIER, 2004, 23), étant donné la proximité géographique et culturelle de la Belgique avec les pays méditerranéens : il faut savoir que d'une part, entre le seizième et le début du dixhuitième siècle, une grande partie de la Belgique est sous la domination de la monarchie espagnole et que, d'autre part, après la signature du protocole italo-belge en 1946, près d'un demi-million d'Italiens viennent travailler dans les mines belges ${ }^{1}$. Toutefois, cette proximité doit aussi être relativisée : à la fin des années 1960, la pizza et la paella sont encore vues comme des plats exotiques (RAULIN, 2000, 30).

Mon intervention est fondée sur des données d'enquêtes ethnographiques effectuées dans différents quartiers bruxellois entre 2009 et 2014. Le but est de montrer comment la troisième révolution consumériste engendre deux paradoxes : d'une part, elle finit par rendre familier et ordinaire ce qui avait été, au premier abord, exotique ou inhabituel; et, d'autre part, si la nourriture acquiert une nouvelle fonction, celle de dépayser, elle sert aussi à baliser une identité "territorialisée ", avec ses spécialités nationales et régionales. Le premier paradoxe conduit à une recherche incessante de nouveautés, leur pouvoir d'attraction dépendant de leur résistance à la banalisation et à un usage généralisé ; et le deuxième à une globalisation grandissante de certains produits locaux et régionaux qui deviennent globaux, parce qu'ils sont porteurs de la valeur "territorialisée » qu'ils incorporent. Résultat inattendu, le principe du cosmopolitisme finit par imposer une logique identitaire et exclusive : les différents commerces parviennent à diversifier le paysage gastronomique, parce que leurs propriétaires sont perçus comme les représentants d'une culture nationale (unique en son genre, car supposée distincte des traditions culinaires des peuples voisins) et d'un terroir - chaque terroir ayant sa propre production, plus ou moins protégée selon les cas, mais toujours considérée comme inimitable et exclusive. En effet, afin de favoriser une politique agricole commune, le règlement européen de 1992 a donné naissance à l'appellation d'origine protégée et à l'indication géographique protégée. Celles-ci instaurent une relation entre le produit et son espace de production qui implique «la non-reproductibilité » et « la mise en place d'une zone délimitée, hors de laquelle la fabrication du produit considéré est juridiquement impossible " (FRAYSSIGNES, 2007, 148).

4 Comme nous le verrons, si pour la première génération de commerçants grecs qui voulaient initier les clients belges à "une cuisine de l'ailleurs", l'exotisme était synchronique, pour la deuxième génération, l'exotisme est diachronique : l'accent est maintenant mis sur la résurgence de plats et de produits du terroir (POULAIN, 2002, 187) c'est-à-dire sur la valeur «territorialisée » que ces produits non industrialisés incorporent. L'exotisme synchronique proposait une cuisine étrangère et dépaysante, l'exotisme diachronique invite à retrouver le bon goût du passé. Si l'industrialisation grandissante de la sphère alimentaire marque les initiatives de la première génération, pour les nouveaux entrants dans le champ, c'est la promesse d'une cuisine authentique et non industrialisée qui prévaut. Cette nouvelle tendance s'inscrit dans l'engouement actuel pour les produits «bio » et la nostalgie pour la pureté de la campagne et de ses produits; elle est aussi liée à la récente valorisation du régime alimentaire méditerranéen, qui fut longtemps considéré comme grossier et carencé avant d'être 
érigé en modèle gastronomique dans les années 1980 (rappelons que ce régime a été inscrit en 2010 par l'Unesco au patrimoine culturel immatériel de l'humanité). Afin de mieux saisir ce processus de réinterprétations et de requalifications, je m'appuierai sur la notion de champ développée par Pierre Bourdieu.

\section{La première génération: les « missionnaires »}

5 Voilà comment, lors d'une interview réalisée le 13 juin 2013, une Grecque qui est arrivée à Bruxelles dans les années 1960, après son mariage à l'âge de dix-neuf ans avec un mineur qui s'est vite orienté vers le domaine des épiceries, m'a décrit le processus d'évolution des habitudes alimentaires belges :

À l'époque, il n'y avait que des produits belges, on ne trouvait pas de produits grecs même pour nous, pour manger. Nous étions les premiers à faire venir des produits grecs. Au début, on les donnait aux Belges gratuitement, pour qu'ils goûtent, et ils ne voulaient même pas goûter. Mais petit à petit, ils les ont appris, la feta, le tarama, les dolmas. Maintenant, ils les connaissent mieux que nous. [...] Nous étions

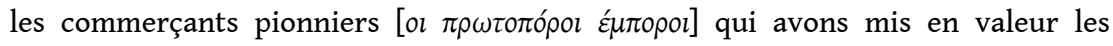
produits grecs.

6 Selon son témoignage, en l'espace d'une cinquantaine d'années, les Belges sont parvenus à mieux connaître les produits grecs que les Grecs eux-mêmes; mais leur apprentissage n'est pas le résultat de leur curiosité puisqu'au début, ils refusaient même de goûter ce qui leur était offert. Son récit n'attribue donc pas la consommation de produits exotiques (en l'occurrence, grecs) au mode de vie ou aux stratégies de distinction des consommateurs. Les protagonistes de ce processus ne sont pas les acheteurs, mais les commerçants, ces initiateurs de goûts nouveaux qui ont continué de pratiquer la familiarisation par l'essai, malgré les hésitations initiales de leurs clients. Mon interlocutrice met donc plus l'accent sur le pouvoir de persuasion et l'insistance des commerçants grecs que sur l'esprit de découverte dont les consommateurs belges auraient pu faire preuve : ce sont les commerçants qui ont su transformer la réticence initiale en attraction.

7 Le commerce de gros de produits grecs vers la Belgique s'organise à partir des années 1970. Selon un grossiste qui a ouvert son magasin dans un quartier bruxellois commerçant en janvier 1983, mais qui collaborait avec les épiciers grecs de Bruxelles depuis 1969,

dans les années 1970, l'État grec aidait ceux qui faisaient des exportations: ils recevaient alors une prime de $10 \%$. Parce que nous étions des missionnaires

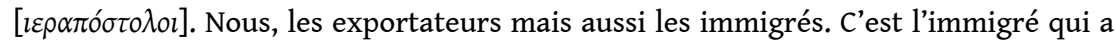
amené ici la civilisation grecque et les produits grecs.

Ce n'est qu'après l'entrée de la Grèce dans la Communauté économique européenne en 1981 que cette politique étatique favorisant les exportations au sein de l'Europe a pris fin.

9 Au tout début, le commerce grec le plus répandu est le café, où l'on sert de l'alcool accompagné d'amuse-gueules. Plusieurs restaurants à Bruxelles ont commencé ainsi, en étoffant progressivement leur offre jusqu'à proposer un véritable menu. Mais nous sommes encore dans la logique de la niche ethnique : ce sont exclusivement les Grecs qui fréquentent ces établissements. Pendant cette période-charnière, le patrimoine culinaire grec, qui consiste en un modèle alimentaire transmis d'une génération à l'autre à l'intérieur de la maisonnée, prend une nouvelle forme, commerciale cette fois. 
Il faut trouver les bons arguments pour attirer une clientèle extérieure au groupe de référence : celui qui est à l'origine de l'offre doit persuader l'acheteur potentiel qu'il peut lui apporter un peu de couleur locale et des sensations nouvelles venues d'ailleurs mais consommées chez soi (SERAÏDARI, 2012). Comme le montre le témoignage précédant, le commerçant grec doit être un « missionnaire » qui prêche les qualités de cette cuisine. Et voilà que son travail est facilité par un allié inattendu: l'industrie cinématographique. Les restaurateurs grecs aiment souligner l'enthousiasme provoqué par la sortie du film Jamais le dimanche le $1^{\mathrm{er}}$ octobre 1960, où l'on entendait Melina Merkouri chanter les Enfants du Pirée, puis par le succès mondial du film Zorba le Grec réalisé en 1964. Le fait que ces images cinématographiques transforment la Grèce en une destination touristique à la mode a une répercussion sur les restaurants grecs, puisqu'à leur retour les Belges essaient de retrouver l'ambiance et les goûts découverts lors des vacances estivales; ils veulent surtout danser le sirtaki, comme plusieurs restaurateurs me l'ont répété : "Tous les restaurants grecs avaient à l'époque cette réputation, de s'amuser [ $v \alpha$ kávovv $\kappa \varepsilon ́ \varphi l]$, de danser sur les tables au son du bouzouki, de casser des assiettes ». Puis, la Grèce antique est, à son tour, invitée à table. Les fresques antiquisantes et les colonnes qui décorent la salle des commerces grecs ${ }^{3}$ sont en concordance avec des noms de plats et de desserts. Par exemple, la carte du Zorba II, un restaurant grec à Bruxelles, proposait des glaces dont les noms évoquaient des dieux antiques: "La troisième jambe d'Apollon » et «Le nectar d'Aphrodite » (menu, sans indication chronologique, publié par GEORGOPOULOS, 2008, 212). Sur le même menu, un peu plus bas, il est noté que «Zorba vous remercie de vous être assis à sa table et d'avoir partagé son repas». Cette phrase ne montre pas seulement que les images cinématographiques et les références antiquisantes coexistent sans aucun problème ; elle suggère aussi un processus d'identification entre le personnage de Zorba, le propriétaire du restaurant (qui vous accueille à sa table) et le client (qui partage le repas de Zorba, voire qui devient Zorba en fréquentant ce restaurant).

10 En effet, tout se passe comme si le fait de manger dans un restaurant grec activait une machine à remonter le temps et à modifier l'espace, comme si ces initiatives marchandes étaient investies d'une valeur patrimoniale dépassant le seul domaine de l'alimentation: les commerçants grecs ne vendaient pas seulement une glace, mais une glace qui faisait apparaître Apollon en plein centre de Bruxelles. Si aujourd'hui ces lieux semblent folkloriques à nos yeux désabusés, plusieurs informateurs ont souligné l'étonnement et l'admiration que les premiers (et les plus soignés, sans doute) de ces restaurants grecs provoquaient chez la clientèle belge: le fait de les fréquenter correspondait à une "expérience culturelle ", certains d'entre eux étaient comme des " cafés littéraires » où ceux qui avaient fait des études classiques pouvaient échanger et passer un moment agréable; dans ces établissements, on ne cassait pas d'assiettes, ou seulement de temps en temps pour amuser quelque touriste égaré. Même pour les clients les moins éduqués, le fait de consommer dans ce cadre devenait un signe de familiarité avec la culture urbaine des classes supérieures.

\section{La nouvelle génération : les « ambassadeurs »}

11 Après l'entrée de la Grèce dans la Communauté économique européenne, une nouvelle vague migratoire arrive à Bruxelles. Ceux qui sont embauchés dans les institutions européennes ne se considèrent pas comme des immigrés, mais comme des expatriés. 
Entre les deux groupes, les contacts sont rares et les passerelles manquent (SERAïDARI, 2012). Installés dans les quartiers populaires et ouvriers de la ville, les immigrés grecs des années 1950 désignent les nouveaux arrivés comme étant des "émigrés de luxe » ou des "mineurs de diamants " [ $\alpha \delta \alpha \mu \alpha v \tau \omega \rho$ púxol], par opposition à eux-mêmes, les mineurs de charbon. Souvent d'origine urbaine et sensiblement plus cultivés et qualifiés, ceux qui sont désignés comme " eurocrates » critiquent à leur tour, la ruralité et les manières rustres de ceux qui jusque-là représentaient la culture grecque en Belgique. Ils évoquent même à leur égard la théorie des « $3 \mathrm{~S}$ » : « souvlaki, sirtaki et

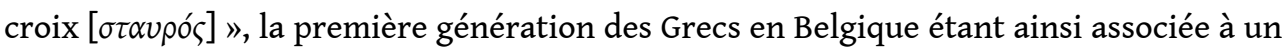
plat emblématique, à une danse rendue célèbre par le cinéma et à une piété caractérisée comme superstitieuse ou excessive.

12 Il faut redonner la parole à la première épicière afin de voir comment les commerçants de la première génération ont été sollicités pour fêter l'heureux événement de 1981, avant de tomber progressivement en disgrâce :

Quand la Grèce est entrée dans l'Union européenne, ils ont fait un marché [ $\varepsilon i ́ x \alpha v$ $\lambda \alpha \ddot{i k n ́}$ dans la rue Archimède. J'ai fait du tarama pendant un mois pour que tous les étrangers puissent en goûter, le mixeur marchait de six heures du matin jusqu'à dix heures du soir. Maintenant, ils ne nous invitent plus nulle part, plus rien [ó $\alpha$

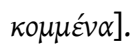

Selon la notion de champ de Pierre Bourdieu 4 , si les "pionniers » développent des stratégies de conservation de leur position dominante dans un champ, les nouveaux entrants sont enclins à des stratégies de subversion et de ruptures critiques :

Les stratégies des nouveaux venus, qui sont aussi les plus jeunes, tendent à rejeter vers le passé les plus anciens et ceux-ci collaborent à la translation du champ qui aboutira à leur déclassement [...] par les stratégies qu'ils mettent en œuvre pour s'assurer la position dominante, la plus proche aussi du déclin.

(BOURDIEU \& DELSAUT, 1975, 15)

14 Depuis les années 1990, la nouvelle génération des restaurateurs grecs se positionne fermement contre la tradition d'un décor antiquisant, perçu de plus en plus comme kitch, et d'une certaine manière de faire la fête (danser le sirtaki et casser des assiettes). Le positionnement de ces nouveaux acteurs, qui revendiquent la création d'une cuisine grecque "post-moussaka ", instaure trois formes de rupture (SERAÏDARI, 2014):

- rupture gustative, leur cuisine étant variée, sophistiquée et inventive, contrairement à la standardisation apportée par ceux qui les ont précédés ;

- rupture spatiale, leurs établissements étant situés dans les quartiers historiques ou aisés, et non dans les quartiers populaires ;

- rupture scénographique : décoration minimaliste et dépouillé versus mauvais goût et clichés folkloriques.

Tandis que la première génération a adapté son offre à la diachronie du repas français (entrée, plat principal, dessert), certains représentants de la nouvelle génération construisent leur carte autour de la notion du froid et du chaud (mezzés chauds ou froids) et accentuent le caractère synchronique de leur offre : le concept du mezzé, qui suggère le partage convivial et le fait de picorer dans une assiette commune, revient maintenant à la mode.

Un article de presse de $2001^{5}$, intitulé « La fin du sirtaki. Oubliez tout ce que vous croyez savoir sur la cuisine hellénique. Aujourd'hui, les restos grecs affichent un nouveau 
profil ", salue le renouveau et ceux qui sont à la recherche de la véritable gastronomie hellénique. Selon ce texte du journal Le Soir, finis les "fast-food» de la première génération, avec leurs «pseudo-colonnades doriques » : « Aux yeux de nombreux Grecs eux-mêmes, il n'y avait pas de feuille de vigne assez grande pour masquer cette trahison gastronomique ». Non seulement la presse belge, mais aussi les journaux grecs, figurent parmi les acteurs impliqués dans ce processus de requalification. En 2009, un des plus grands journaux grecs, Eleftherotypia, a décerné pour la quatrième année consécutive les Prix Gourmet : dans ce cadre, un restaurateur grec de Belgique a été nommé "Meilleur ambassadeur de la cuisine grecque dans le monde ». La presse belge l'a présenté au public en insistant sur sa recherche, depuis les années 1980, des pionniers de l'agriculture biologique en Grèce et des produits des moines du Mont Athos (miel, vin, huile d'olive). Cette «cuisine d'auteur», que la nouvelle génération revendique, est fondée sur la préférence pour des produits locaux, comme les favas de Santorin ou l'avgotaracho de Missolonghi (présenté comme le «caviar grec $»)^{6}$.

Selon notre premier témoignage, les Belges connaissent maintenant mieux la feta, le tarama et les dolmas que les Grecs eux-mêmes. La familiarisation grandissante a certainement changé le statut de ces produits: la feta est aujourd'hui moins un produit-phare de la cuisine grecque qu'un produit transculturel, comme le montre la bataille entre l'État grec et les autres États membres de l'Union européenne sur la protection de la dénomination «feta» (PETRIDOU, 2005). C'est pour cette raison que la nouvelle génération de commerçants grecs met en avant des produits qui ne sont pas encore devenus transculturels. Par leurs références géographiques et dialectales, ces aliments s'inscrivent dans une tendance plus générale, celle d'une nouvelle construction de la ruralité qui conduit à l'exoticisation du familier (YIAKOUMAKI, 2006). En effet, depuis quelques décennies, les produits biologiques, qui sont liés au souci environnemental, à la biodiversité, au terroir et ses spécificités, au respect des saisons et à la consommation responsable, occupent une place grandissante dans le domaine de l'alimentation.

L'exotisme diachronique qui caractérise la période actuelle est marqué par la (re)découverte des produits qui avaient été malmenés par l'industrialisation de l'agroalimentaire. Il suffit de prendre l'exemple du tarama pour mieux comprendre comment le client est invité à sortir des sentiers battus : si dans les commerces de la première génération, celui-ci est fluorescent ou " rose pétant », les nouveaux entrants proposent un tarama méconnaissable qui est blanc-beige naturel, car exempt de colorants.

19 Si la première génération a joué le rôle de vulgarisateurs, selon la théorie du champ de Bourdieu, les nouveaux entrants insistent sur la singularité afin d'arracher le champ à l'immobilité et la banalisation; leur présence contribue à réparer la dévaluation entraînée par l'accroissement du nombre des consommateurs de la cuisine grecque. Dans le même temps, ils poussent vers une plus grande professionnalisation, dans la mesure où la première génération était composée d'autodidactes sans diplôme hôtelier. Les qualifications et les titres des nouveaux entrants vont de pair avec leur aspiration à la reconnaissance gastronomique : «C'est en produisant la rareté du producteur que le champ de production symbolique produit la rareté du produit» (BOURDIEU \& DELSAUT, 1975, 21). En opposant à l'uniformité élémentaire et au patrimoine «mineur » des premiers commerçants le patrimoine «majeur » et inventif que la nouvelle génération 
a construit à partir des années 1980 , cette dernière introduit ainsi un principe de dualisation à l'intérieur de ce patrimoine culinaire.

\section{Les effets de la crise économique}

20

Avec la crise économique qui frappe la Grèce depuis 2010, apparait une nouvelle vague d'immigration grecque vers la Belgique. Ce n'est pas un hasard si certains restaurants qui ouvrent après cette date vendent des produits artisanaux que le chef de l'établissement utilise pour élaborer son menu : cette offre les transforme en une sorte d'« épicerie fine ", où les convives, à la fin du repas, peuvent acheter les produits qui ont donné une saveur particulière aux plats qu'ils viennent de déguster. Ces "restaurants-épiceries fines ", qui connaissent un essor grandissant, sont aussi liés à la crise, dans la mesure où leurs propriétaires considèrent qu'ils aident, de cette manière, les petites entreprises et les producteurs grecs à se faire connaître à l'étranger, ce qui est essentiel pour leur survie par les temps actuels. Ils se dotent ainsi d'une mission sociale qui les différencie par rapport à la deuxième génération qui a voulu, d'une part, satisfaire la nouvelle clientèle des eurocrates grecs qui se sont installés à Bruxelles dans les années 1980 ; et d'autre part, construire une nouvelle image de la cuisine grecque. La propriétaire d'un de ces «restaurants-épiceries fines» qui a ouvert en novembre 2013 dans un quartier aisé d'Uccle, a longuement insisté, dans son interview,

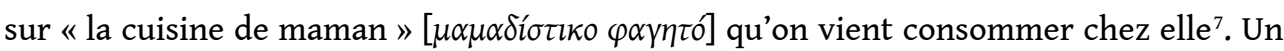
des ingrédients qui définit ses entrées est le petimezi, le moût, qu'elle présente comme étant « un caramel naturel » idéal pour assaisonner les salades; le client peut acheter de petites bouteilles de petimezi sur place. Néanmoins, dans la cuisine traditionnelle grecque, le petimezi n'a jamais joué ce rôle. Le produit est certes traditionnel, mais son utilisation est nouvelle et détournée: c'est ce décalage qui caractérise l'inventivité " post-moderne » de la nouvelle génération.

$\mathrm{Si}$, tant en Grèce qu'en Belgique, le vinaigre balsamique est devenu, depuis environ vingt ans, un ingrédient communément utilisé pour la préparation des salades, le petimezi pourrait progressivement l'évincer, grâce à ce type de restaurants qui attribuent de nouvelles fonctions culinaires à des produits grecs encore peu connus à l'étranger. Étant donné que les premiers commerçants grecs ont réussi à familiariser les Belges avec des produits grecs devenus aujourd'hui transculturels, le processus ne semble pas être particulièrement nouveau. Ce qui est nouveau, en revanche, est le fait que la nouvelle utilisation du petimezi dans les salades constitue une tendance qui se développe, pour la première fois, de manière parallèle et simultanée en Belgique et en Grèce. Bien évidemment, le niveau et le caractère de l'exotisme varient entre le pays de référence (la Grèce) et le pays d'accueil (la Belgique, où les commerçants grecs développent leurs activités): en Belgique, il s'agit de l'introduction d'un nouveau produit « exotique » tout court; en Grèce, cette évolution n'entre pas dans la catégorie de l'exotisme diachronique (le petimezi étant uniquement associé aux desserts selon les recettes traditionnelles), mais dans celle de l'exoticisation du familier'.

23 En effet, la nouvelle génération des commerçants grecs en Belgique puise ses idées novatrices dans deux registres distincts : l'exotisme diachronique et l'exoticisation du familier. En revanche, comme nous l'avons vu, la première génération a plutôt joué la carte d'un exotisme synchronique, en familiarisant les Belges avec "une cuisine de l'ailleurs» et ses produits; toutefois, la dimension diachronique était également 
présente dans ses initiatives marchandes, dans la mesure où la consommation de plats grecs était censée transporter les clients à l'époque de la Grèce antique.

\section{BIBLIOGRAPHIE}

BOURDIEU Pierre \& DELSAUT Yvette, 1975, « Le couturier et sa griffe : contribution à une théorie de la magie », Actes de la recherche en sciences sociales, vol. 1, $\mathrm{n}^{\circ} 1, \mathrm{p} .7-36$.

ÉLOIRE Fabien, 2011, "Capital social et concurrence de statut », Revue européenne des sciences sociales $n^{\circ} 49,2$, p. 7-41.

FRAYSSIGNES Julien, 2007, « L'AOC Roquefort : une filière emblématique », CIHEAM, MediTERRA 2007, Presses de Sciences Po « Annuels », p. 147-184.

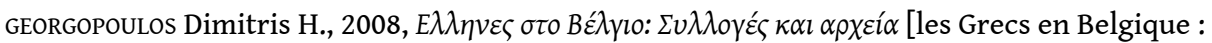
collections et archives], Bruxelles : Adelfoi Kyriakidi (en grec).

PETRIDOU Elia, 2005, « Au pays de la feta : négociation de la grécité dans le contexte européen », Ethnologie française Xxxv, 2, p. 255-265.

POULAIN Jean-Pierre, 2002, Sociologies de l'alimentation : les mangeurs et l'espace social alimentaire, Paris : Presses Universitaires de France.

RAULIN Anne, 2000, l'Ethnique est quotidien : diasporas, marchés et cultures métropolitaines, Paris :

L'Harmattan.

RÉGNIER Faustine, 2004, l'Exotisme culinaire : essai sur les saveurs de l'Autre, Paris : Presses Universitaires de France.

SERAÏDARI Katerina, 2012, la Ville, la Nation et l'Immigré : rapports entre Grecs et Turcs à Bruxelles, Paris : L'Harmattan.

SERAÏDARI Katerina, 2012, « Paysages marchands en contexte migratoire : les objets dans les commerces grecs et turcs de Bruxelles », Études balkaniques. Cahiers Pierre Belon n ${ }^{\circ} 18$, p. 161-178.

SERAÏDARI Katerina, 2014, « Restaurants grecs et turcs à Bruxelles : concurrence ou émulation? », Ethnologie française XLIV, 1, p. 115-124.

YIAKOUMAKI Vassiliki, 2006, “'Local', 'ethnic' and 'rural' food: On the emergence of 'cultural diversity' in Post-EU-Accession Greece”, Journal of Modern Greek Studies, vol. 24, pp. 415-445.

\section{NOTES}

1. Certains mineurs grecs qui côtoient leurs homologues italiens s'habituent progressivement à la consommation des produits italiens, et, quand leur famille vient les rejoindre en Belgique, l'initient à ces nouveaux goûts : jambon de Parme, parmesan, mozzarella. Par exemple, un couple âgé d'informateurs grecs a gardé l'habitude, depuis les années 1960, d'acheter un panettone à Noël, conformément à la tradition italienne. 
2. Cette inscription trouve sa justification au-delà de la sphère alimentaire : la diète méditerranéenne est saluée non seulement pour son équilibre, mais aussi pour les valeurs de l'hospitalité, du bon voisinage et du dialogue interculturel qu'elle véhicule.

3. Sur la manière dont les commerces grecs, mais aussi turcs, sont décorés à Bruxelles (utilisation de drapeaux nationaux et d'objets religieux, mémoriels et folkloriques), voir SERAÏDARI (2012).

4. Pour une utilisation de cette théorie dans le cadre d'une recherche sur les propriétaires de restaurants installés dans l'agglomération lilloise, voir ÉLOIRE Fabien (2011).

5. Publié dans Le Soir, vendredi 27 juillet 2001, p. 20 et signé par Michel Verlinden.

6. Même en Grèce, ce mets régional n'a jamais connu une diffusion commerciale et une consommation importante, à cause de son prix élevé et d'un volume de production réduit.

7. Dans les restaurants grecs de Bruxelles, l'exotisme diachronique se présente, d'un côté, sous la forme d'une nourriture « fait maison » (comme ici), capable de ressusciter des souvenirs de l'enfance par sa simplicité et sa fraîcheur - et cela, malgré les différences entre les cuisines grecque et belge; et de l'autre côté, sous la forme d'un retour au passé préindustriel. Dans ce cas, l'exotisme diachronique n'insiste pas sur la valeur «territorialisée » et unique du terroir : un bon produit grec évoque la «cuisine de maman » de la même manière qu'un bon produit belge le fait (même si les Belges ne mangeaient pas de plats grecs dans leur enfance).

8. La bande dessinée les Vieux fourneaux. Bonny et Pierrot de Wilfrid LUPANO et Paul CAUET (Dargaud Benelux, 2014) montre bien comment la baguette est devenue un terme qui ne signifie plus rien dans une boulangerie française vendant des produits qui exoticisent le familier, comme la Sarmentine, la Grand Siècle, la Câlinette, l'Origine, l'Essentielle et d'autres marques déposées de ce type.

\section{RÉSUMÉS}

Fondé sur des données ethnographiques recueillies depuis 2009, cet article analyse les représentations, les activités commerciales et les pratiques alimentaires des migrants grecs à Bruxelles. Les Grecs ont contribué à développer et réorienter les choix alimentaires et le goût de leur clientèle à laquelle ils proposent des produits qui paraissent exotiques avant de devenir, progressivement, des éléments à part entière de la gastronomie locale. En s'opposant à cette tendance de banalisation, la nouvelle génération essaie de réinventer la cuisine grecque et de remplacer des produits grecs devenus transculturels par d'autres éléments, moins connus et moins industrialisés.

Based on ethnographical data collected since 2009, this article analyzes the representations, the commercial activities and the food practices of Greek migrants in Brussels. Greeks have contributed to develop and to re-orient the food choices and the taste of their clientele, to whom they propose products that seemed exotic before progressively becoming part of the local 
gastronomy. Through its opposition to this tendency to banalization, the new generation tries to reinvent Greek cuisine and to replace Greek products that became transcultural by other elements, less known and less industrialized.

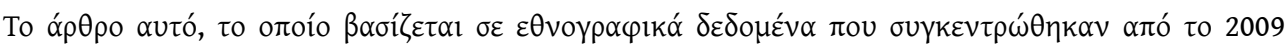

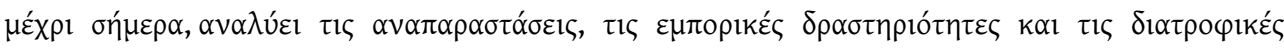

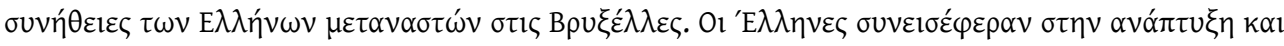

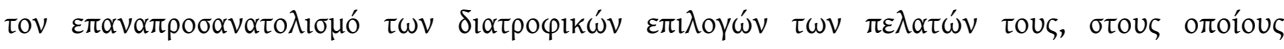

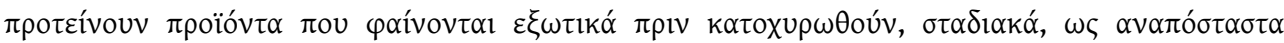

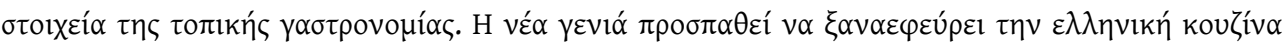

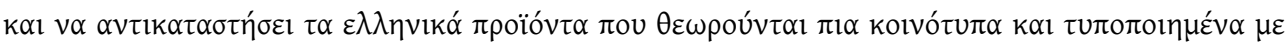

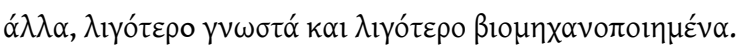

\section{INDEX}

Index géographique : Grèce

Mots-clés : nourriture, nourriture, produits bio, produits bio, transferts culturels, transferts culturels, immigration grecque, immigration grecque

Thèmes : Histoire sociale, Histoire culturelle

motsclestr Yiyecek, Bio ürünleri, Kültürel transferler, Yunan Göç, Belçika, yirminci yüzyıl, Yirmi birinci yüzyll, Sosyal tarih, Kültürel tarih

Keywords : Food, Bioproducts, Cultural transfers, Greek immigration, Belgium, Twentieth Century, Twenty First century, Social history, Cultural history

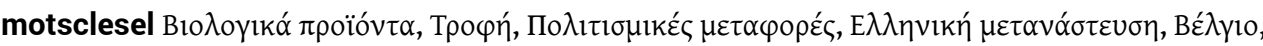

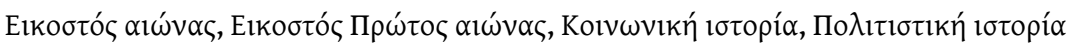
motsclesmk ХРАНА, ПРОИЗВОДИТЕЛЬ, КУЛТУРНИ ТРАНСФЕРИ, ГРЧКИОТ ИМИГРАЦИЈА, БЕЛГИЈА, ДВАЕСЕТТИОТ ВЕК, ДВАЕСЕТ И ПРВИОТ ВЕК, СОЦИЈАЛНА ИСТОРИЈА, КУЛТУРНА ИСТОРИЈА

Index chronologique : vingtième siècle

\section{AUTEUR}

\section{KATERINA SERAÏDARI}

LISST, Toulouse 